\title{
Analyse quantitative des uréides glyoxyliques dans les sèves et les extraits végétaux
}

\author{
Claudette MONGET \& Claude TENDILLE \\ I.N.R.A., Laboratoire du Métabolisme et de la Nutrition des Plantes, Centre de Recherches de Versailles, \\ route de St-Cyr, F 78000 Versailles
}

Mots clés additionnels : Allantoïne, acide allantoïque, extraction et dosage.

The numerous classical methods used for determining glyoxylic ureides are primarily concerned with determination itself, but rarely take into account the collection and processing of samples. A comprehensive method of determining these compounds in plant tissues and exuded sap has been proposed. The processes of taking samples is precisely described and changes in the techniques of glyoxylic ureide hydrolysis and determination are proposed.

Additional key words : Allantoin, allantoic acid, extraction and determination.

\section{INTRODUCTION}

Chez les végétaux, les uréides glyoxyliques, allantoïne et acide allantoïque, sont des composés azotés d'accumulation universellement répandus. Ils peuvent être transportés dans divers organes par la sève du xylème pour être utilisés dans de nouvelles synthèses. Chez les légumineuses, ces substances sont en outre impliquées dans le transport, de l'azote réduit par les bactéries symbiotiques des nodosités (JOLIVET \& MOSSÉ, 1982 ; PATTERSON et al., 1982 ; VAN BERKUM \& SLOGER, 1963 ; ZENGBE, 1984).

Les études consacrées aux uréides glyoxyliques connaissent un regain d'intérêt, non seulement chez les légumineuses, mais aussi chez les non légumineuses ; elles ont permis, en particulier, de mettre en évidence la présence d'acide allantoïque dans la sève racinaire de concombre et d'allantoïne dans la sève racinaire de tomate (JOLIVET et al., 1985 ; JOLIVET et al., 1986).

Ces recherches rendent nécessaire l'utilisation de techniques d'extraction et d'analyse précises, rapides, reproductives et peu onéreuses. L'étude bibliographique du sujet apporte peu de renseignements sur les techniques d'extraction, du fait que les dosages sont en général effectués dans des liquides biologiques (urine, sang...). En revanche, les méthodes de dosages sont nombreuses et leur diversité est symptomatique des difficultés rencontrées par les analystes. En effet, il est possible de déterminer les uréides glyoxyliques, soit en dosant les molécules formées lors de leur dégradation par hydrolyse, soit en dosant directement par chromatographie les molécules d'allantoïne et d'acide allantoïque. Il est connu qu'une hydrolyse basique transforme l'allantoïne en acide allantoïque et qu'une hydrolyse acide est utilisée ensuite pour scinder la molécule d'acide allantoïque en une molécule d'acide glyoxylique et 2 molécules d'urée (fig. 1).

Les méthodes habituellement utilisées pour doser les uréides glyoxyliques sont résumées dans le tableau 1.

Après avoir essayé les méthodes utilisées par YOUNG \& CONWAY (1942), VOGELS \& VAN DER DRIFT (1970), PATTERSON et al. (1982), nous avons retenu celle de VOGELS \& VAN DER DRIFT (1970) en raison de sa simplicité et de son efficacité. Cependant, à l'usage, nous avons constaté qu'il était nécessaire de modifier certains points de cette technique. La présente note décrit 
<smiles>COC(=O)NC(NC(N)=O)C(N)=O</smiles><smiles>NC(=O)[OH+]C(N)=O</smiles>

Figure 1

Transformation de l'allantoïne (I) en acide allantoique (2) puis en 2 molécules d'urée (3) et une molécule d'acide glyoxylique (4).

Conversion of allantoin (1) into allantoic acid (2) then into 2 moles of urea (3) and one mole of glyoxylic acid (4).

\section{TABLEAU}

Méthodes habituelles de dosage des uréides glyoxiliques.

Usual methods for glyoxylic ureide determination.

I - Méthodes basées sur le dosage des produits obtenus après hydrolyses.

1. Urée.

a. Méthode pondérale après action de l'uréc sur le xanthydrol (Fosse \& BossuYT, 1927).

b. Méthode colorimétrique après obtention d'un indophénol (Domnas, 1961 ; Patton \& Crouch, 1977 ; Patterson et al., 1982).

2. Acide glyoxylique.

Méthode colorimétrique de Rimini-Schryver (Fosse \& HieulLe, 1924).

II - Méthodes d'analyses directes de l'allantö̈ne par chromatographie.

1. Couche mince.

a. Celluiose (ABRAHAm et al., 1976).

b. Gel de silice (VRABSK1 et al., 1978).

2. Haute pression (TIEMEYER \& GieseCKE, 1982).

la méthode que nous proposons pour l'analyse des uréides glyoxyliques. En plus de l'amélioration que nous apportons à la technique de dosage préconisée par VOGELS \& VAN DER DRIFT (1970), nous indiquons les processus de prélèvement des tissus végétaux ou des sèves racinaires et les modes de fixation et d'extraction que nous pratiquons.

\section{EXPOSÉ DE LA MÉTHODE UTILISÉE}

\section{A. Préparation des extraits}

\section{Sève brute exsudée}

Les tiges des plantes sont coupées à quelques centimètres au-dessus du collet. Des tuyaux en caoutchouc souple ou en polyuréthane sont placés immédiatement sur le restant de la tige et serrés à l'aide d'un collier. Le manchon opaque ainsi formé permet d'éliminer l'action de la photosynthèse, même minime, qui pourrait se produire au niveau du tronçon du restant de tige (fig. 2). La sève est récoltée par aspiration à l'aide
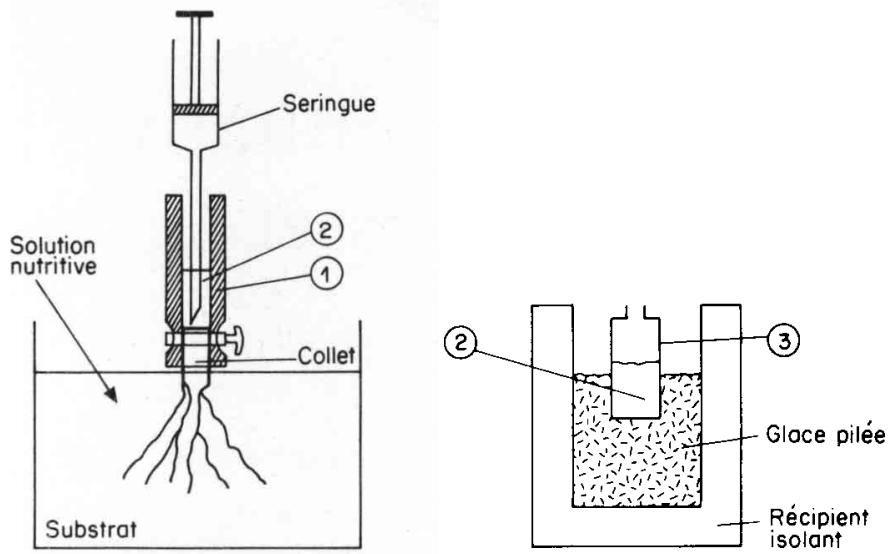

Figure 2

Dispositif expérimental de prélèvement de la sève : (1) manchon souple serré sur la tige ; (2) sève ; (3) récipient contenant la sève recueillie.

Experimental sap sampling device: (1) flexible tube fixed on the stalk with a clamp; (2) sap ; (3) container for collecting sap.

d'une seringue soit, heure par heure, soit globalement durant 5 à 6 heures, maximum. Le volume de sève prélevé est mesuré puis réparti en plusieurs fractions qui sont stockées au congélateur avant d'être prélevées pour analyses.

\section{Tissus végétaux}

\section{a) Préparation du matériel végétal}

Un échantillonnage rigoureux est effectué sur différents organes des plantes, ce qui permet d'obtenir des fractions végétales représentatives. Une fraction sera utilisée pour l'analyse des uréides, et une autre pour la détermination de la masse de substance sèche.

Le tissu végétal est soit analysé immédiatement, soit broyé dans un mortier en présence d'azote liquide, puis lyophilisé ou conservé dans un congélateur à $-20^{\circ} \mathrm{C}$; la masse de substance sèche de l'échantillon lyophilisé est déterminée au moment de l'extraction.

L'extraction de la substance sèche préconisée par PATTERSON et al. (1982) ne peut être conseillée étant donné l'évolution des produits azotés libres durant la dessiccation. Il est préférable, comme nous l'avons toujours fait, d'effectuer l'extraction sur la substance fraîche ou lyophilisée. Une partie aliquote (5 à $10 \mathrm{~g}$ de substance fraîche) est broyée dans un broyeur Sorvall en présence de 100 à $200 \mathrm{ml}$ d'un tampon Tris, $\mathrm{HCl}$ 0,05 $\mathrm{M}$ ajusté à $\mathrm{pH}=7,4$ avec de l'acide chlorhydrique (VAN DER DRIFT \& VOGELS, 1966). L'extrait est filtré sur un fin tissu de nylon et le filtrat conservé une nuit au réfrigérateur avant le dosage.

La filtration de certains extraits qui contiennent de l'amidon est difficile et la présence de fines particules en suspension augmente leur densité optique. Il est donc nécessaire de comparer spectrophotométriquement l'extrait à doser, coloré et trouble, à un " blanc échantillon " contenant les réactifs sauf le ferricyanure de potassium qui permet par oxydation le développement de la coloration rose.

b) Préparation d'une solution standard d'allantoïne

L'allantoïne (origine Sigma) de masse molaire $158 \mathrm{~g}$ est séchée en étuve pour obtenir un produit de réfé- 
rence anhydre. Une quantité $\mathrm{P}$ d'allantoïne anhydre avoisinant $100 \mathrm{mg}$ est pesée puis dissoute dans $50 \mathrm{ml}$ d'eau désionisée. Cette solution mère peut se conserver pendant 1 mois au réfrigérateur. En revanche, la solution utilisée comme standard est préparée extemporanément en diluant $1 \mathrm{ml}$ de cette solution mère dans $100 \mathrm{ml}$ d'eau désionisée. La concentration en nanomoles de cette solution standard est de :

$$
\frac{\mathrm{Pmg}}{158} \times \frac{1}{50} \times \frac{1}{100} \times 10^{6} \text { par } \mathrm{ml}
$$

\section{B. Hydrolyses des extraits}

Dans les sèves et surtout dans les extraits végétaux les uréides peuvent se trouver à l'état de liaisons plus ou moins complexes qui sont plus difficiles à hydrolyser que les corps simples (TRIJBELS \& VOGELS, 1966). Aussi les durées d'hydrolyses préconisées par VoGELS \& VAN DER DRIFT (1970) ont été doublées. Nous avons vérifié que les résultats ne sont pas, de ce fait, perturbés (tabl. 2).

\section{TABLEAU 2}

Quantités d'allantoine (nanomoles) retrouvées en fonction des durées d'hydrolyses basique ( 8 à $16 \mathrm{mn}$ ) puis acide $(4$ à $8 \mathrm{mn})$.

Amounts of allantoin (nanomoles) in relation to basic ( 8 to $10 \mathrm{mn}$ ) and acid (4 to $8 \mathrm{mn}$ ) hydrolysis time.

\begin{tabular}{ccc}
\hline \hline Apportée & $\begin{array}{c}\text { Retrouvée après } \\
8 \mathrm{mn} \text { (hydrolyse basique) } \\
+4 \mathrm{mn} \text { (hydrolyse acide) }\end{array}$ & $\begin{array}{c}\text { Retrouvée après } \\
+4 \mathrm{mn} \text { (hydrolyse basique) }\end{array}$ \\
\hline \multirow{2}{*}{153} & 144 & 148 \\
306 & 254 & 298 \\
\hline \hline
\end{tabular}

\section{Hydrolyse acide}

Une partie aliquote (1 à $4 \mathrm{ml}$ ) de l'extrait végétal, diluée ou non avec de l'eau désionisée pour obtenir un

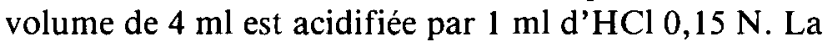
solution acide $(5 \mathrm{ml})$ est chauffée à $100^{\circ} \mathrm{C}$ pendant $8 \mathrm{mn}$ puis refroidie rapidement.

\section{Hydrolyse alcaline suivie d'une hydrolyse acide}

Une partie aliquote (1 à $3 \mathrm{ml})$ de l'extrait végétal est diluée ou non avec de l'eau désionisée pour obtenir un volume final de $3 \mathrm{ml}$. Le milieu alcalinisé par addition de $1 \mathrm{ml}$ de $\mathrm{NaOH} 0,5 \mathrm{~N}$ est chauffé à $100^{\circ} \mathrm{C}$ pendant $16 \mathrm{mn}$ puis refroidi rapidement; il est ensuite acidifié par $1 \mathrm{ml}$ d' $\mathrm{HCl} 0,65 \mathrm{~N}$ et porté à ébullition pendant $8 \mathrm{mn}$ puis refroidi rapidement.

Les hydrolysats sont filtrés et les tubes d'hydrolyse rincés avec du tampon tris $\mathrm{HCl}$ dans des fioles de $25 \mathrm{ml}$, en attente du dosage. Il est très important, après l'hydrolyse acide, d'effectuer immédiatement le dosage de l'acide glyoxylique formé qui est peu stable.

La prise d'essai pour hydrolyse est habituellement de $3 \mathrm{ml}$ mais, dans le cas d'échantillons ne contenant que de faibles quantités d'uréides, l'utilisation d'une prise plus importante devient nécessaire, ce qui a pour conséquence de modifier les conditions de dilution lors des hydrolyses. Nous avons vérifié, à différentes con- centrations de la solution standard d'allantoïne, que les quantités d'allantoïne retrouvées ne sont pas ou très peu influencées par l'utilisation d'un volume plus important d'échantillon, ce volume pouvant atteindre $6 \mathrm{ml}$ (tabl. 3).

\section{TABLEAU 3}

Quantités d'allantoïne (nanomoles) retrouvées en fonction du volume de la prise d'essai avant hydrolyse. Amounts of allantoin (nanomoles) in relation to sample volume before hydrolysis.

\begin{tabular}{ccc}
\hline \hline & \multicolumn{2}{c}{ Retrouvée après hydrolyses dans } \\
\cline { 2 - 3 } & $3 \mathrm{ml}$ & $6 \mathrm{ml}$ \\
\hline 158 & 140 & 160 \\
316 & 288 & 298 \\
474 & 440 & 480 \\
\hline
\end{tabular}

\section{Dosage des uréides glyoxyliques}

$\mathrm{Au}$ cours de la réaction colorée de RIMINI-SCHRYVER, le glyoxylate réagit avec la phénylhydrazine $\mathrm{C}_{6} \mathrm{H}_{5}-\mathrm{NH}-\mathrm{NH}_{2}$ pour former une phénylhydrazone qui est oxydée par le ferricyanure de potassium $\mathrm{K}_{3} \mathrm{Fe}(\mathrm{CN})_{6}$, en présence d'un acide fort, pour produire un chromophore rouge " fuchsine " intense, peu stable, présentant un maximum d'absorption à $520 \mathrm{~nm}$ (fig. 3).

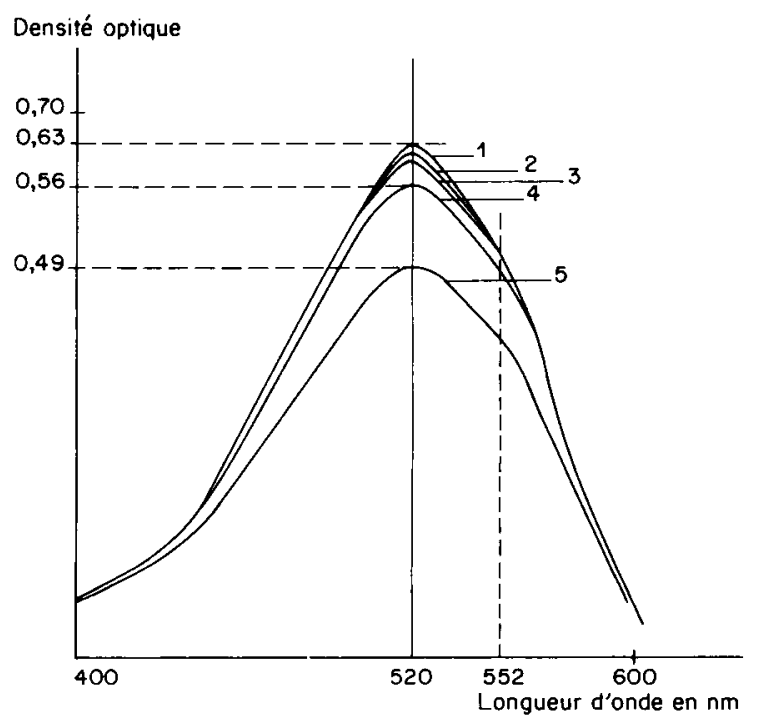

Figure 3

Spectre d'absorption en fonction du temps du 1,5-diphénylformazan et détermination de la stabilité de ce composé.

Absorption pattern of 1,5-diphenylformazan with time and determination of its stability.

Selon KRAMER et al. (1959), ce produit coloré serait le 1,5-diphénylformazan, 3-carboxylique, mais d'après MATSUI et al. (1965) il se forme en fait un 1,5-diphénylformazan.

\section{Formation de la phénylhydrazone}

Dans la fiole jaugée, $1 \mathrm{ml}$ de solution de phénylhydrazine, $\mathrm{HCl}$ et $1 \mathrm{ml}$ de tampon Tris, $\mathrm{HCl}$ à $\mathrm{pH}=7,4$ 
sont ajoutés. Il se forme alors une phénylhydrazone de l'acide glyoxylique. La solution de phénylhydrazine (100 $\mathrm{mg}$ dans $30 \mathrm{ml}$ d'eau désionisée) est préparée journellement.

La formation de la phénylhydrazone a été étudiée par VOGELS \& VAN DER DRIFT (1970). Ils ont montré que le $\mathrm{pH}$, la concentration en phénylhydrazine sont des facteurs importants et que la formation de la phénylhydrazone est complète en $5 \mathrm{mn}$. Cependant, nous avons constaté que la réaction de formation de la phénylhydrazone dure effectivement $10 \mathrm{mn}$ environ (tabl. 4). En outre, les ions phosphates utilisés par VOGELS \& VAN DER DRIFT (1970) élèvent le pH au voisinage de 6 et amènent le développement d'une coloration rose parasite qui augmente la densité optique du «blanc» contenant les réactifs. Cette coloration n'apparaît pas si le $\mathrm{pH}$ reste en dessous de 6 .

\section{TABLEAU 4}

Variation observée dans la densité optique à $332,5 \mathrm{~nm}$ de la phénylhydrazone de l'acide glyoxylique en fonction du temps écoulé après addition de la phénylhydrazine- $\mathrm{HCl}$.

Variation in optical density at $332.5 \mathrm{~nm}$ of glyoxylate phenylhydrazone with time after addition of phenylhydrazine- $\mathrm{HCl}$.

\begin{tabular}{rr}
\hline \hline 2 minutes & 0,13 \\
8 minutes & 0,22 \\
10 minutes & 0,24 \\
150 minutes & 0,24 \\
\hline
\end{tabular}

Nous avons remplacé le tampon phosphate par le tampon Tris, $\mathrm{HCl} 0,05 \mathrm{M}$ à $\mathrm{pH}=7,4$ et n'avons observé ni ralentissement de la formation de la phénylhydrazone, ni aucun affaiblissement de l'intensité de la coloration. Dans les 2 cas le $\mathrm{pH}$ après hydrolyse reste aux environs de 3 , ce qui évite la formation de la coloration parasite.

\section{Formation du 1,5-diphénylformazan}

Les mélanges réactionnels sont refroidis pendant $5 \mathrm{mn}$ à $0{ }^{\circ} \mathrm{C}$ en présence de glace pilée. On ajoute $6 \mathrm{ml}$ d' $\mathrm{HCl}$ concentré froid. L'ensemble est agité vigoureusement puis laissé en repos pendant $5 \mathrm{mn}$. On ajoute alors $1 \mathrm{ml}$ d'une solution de ferricyanure de potassium (500 $\mathrm{mg}$ dans $30 \mathrm{ml}$ d'eau) préparée extemporanément. Après agitation et 5 nouvelles minutes d'attente, les mélanges réactionnels sont laissés à la température ambiante. Après $10 \mathrm{mn}$, le volume est ajusté à $25 \mathrm{ml}$ avec de l'eau désionisée et les mesures spectrophotométriques sont effectuées, d'une part, au maximum d'absorption $520 \mathrm{~nm}$ et, d'autre part, à $552 \mathrm{~nm}$ où existe un épaulement caractéristique (fig. 3). La précision des dosages est de l'ordre de 6 à 10 p. 100 .

La formation du 1,5-diphénylformazan est fonction de l'acidité du milieu, de l'oxydation par le ferricyanure de potassium et de la température de la solution lors de la formation du 1,5-diphénylformazan. L'acidité du milieu est particulièrement importante. Il a été vérifié que la quantité d'HCl concentré ajoutée doit être suffisante pour obtenir une densité optique maximum (tabl. 5). Le 1,5-diphénylformazan formé en milieu chlorhydrique suffisamment acide est stable pendant environ $40 \mathrm{mn}$, puis l'intensité de la coloration diminue progressivement (fig. 3). Ces résultats
TABLEAU 5

Influence du volume d'HCl concentré ajouté, sur l'intensité de la coloration du 1,5-diphénylformazan (pour une même quantité d'allantoine).

Influence of the volume of concentrated $\mathrm{HCl}$ supplied on the coloration intensity of the 1,5-diphenvlformazan (for an identical amount of allantoin).

\begin{tabular}{cc}
\hline HCl ajouté $(\mathrm{ml})$ & Densité optique \\
\hline 2 & 0,42 \\
3 & 0,47 \\
5 & 0,61 \\
6 & 0,62 \\
\hline
\end{tabular}

sont en accord avec ceux observés par YOUNG \& CONWAY (1942), c'est-à-dire 8 et 20 p. 100 d'affaiblissement après $1 \mathrm{~h}$ et $2 \mathrm{~h}$ respectivement.

\section{Interférences}

Divers composés chimiques risquent d'interférer lors du dosage des uréides (YounG \& CONWAY, 1942). Ce sont des molécules présentant un groupement $\mathrm{H}-\mathrm{CHO}$ ou des composés puriques précurseurs de l'allantoïne. En pratique, seul l'acide urique risque, dans certains cas, de modifier les résultats des analyses. Les acides cétoniques (pyruvique et cétoglutarique) n'interfèrent pas avec le dosage des uréides (VAN BERKUM \& SLOGER, 1983). La citrulline qui ne libère pas, par hydrolyses, de l'acide glyoxylique ne peut donc être dosée comme uréide.

Des dosages avant hydrolyse ont permis de constater que les extraits dosés ne contiennent pas d'acide glyoxylique susceptible de provoquer une surestimation des uréides.

\section{RÉSULTATS ET DISCUSSION}

La mise en évidence d'une nouvelle forme de transport de l'azote organique, l'acide allantoïque dans la sève racinaire de concombre (JOLIVET et al., 1985) ainsi que la mise en évidence de voies différentes de synthèse des uréides glyoxyliques chez la tomate et le concombre (JOLIVET et al., 1986) ont été obtenues en utilisant cette méthode. D'autres résultats, non publiés, d'analyse des uréides glyoxyliques par cette méthode sont donnés dans le tableau 6 .

\section{TABLEAU 6}

Analyse des uréides glyoxyliques dans les feuilles, les tiges, les racines de jeunes plants de Phaseolus vulgaris.

Quantitative determination of glyoxylic ureides in leaves, stems and roots of young bean plants.

\begin{tabular}{lccc}
\hline $\begin{array}{c}\text { Composés } \\
\text { uréiques en } \\
\text { nmol/g M.F. }\end{array}$ & $\begin{array}{c}\text { Acide } \\
\text { allantoique } \\
+ \text { allantoine }\end{array}$ & $\begin{array}{c}\text { Acide } \\
\text { allantoïque }\end{array}$ & $\begin{array}{c}\text { Allantoïne } \\
\text { (par } \\
\text { différence) }\end{array}$ \\
\hline Feuilles & 333 & 200 & 133 \\
Tiges & 287 & 193 & 94 \\
Racines & 1000 & 778 & 222 \\
& 983 & 775 & 208 \\
& 561 & 396 & 165 \\
\hline
\end{tabular}


Les difficultés inhérentes à la méthode de dosage des uréides glyoxyliques utilisée auparavant apparaissaient surtout lors de leur application à du matériel végétal. Le présent travail a permis de remédier à ces difficultés et d'obtenir des résultats reproductibles grâce à l'augmentation de la durée d'hydrolyse, à l'allongement du temps de formation de la phénylhydrazone, à l'emploi d'un tampon Tris-HCl.

Cependant des composés tels que les uréidoglycolates, peuvent, par dégradation, libérer de l'acide glyoxy- lique. Ces composés n'ont été signalés, jusqu'à présent, que par TRIJBELS \& VOGELS (1966) lors de la dégradation de l'allantoïne par un microorganisme, Pseudomonas acidovorans. Il est toutefois possible qu'il en existe dans des tissus végétaux. Aussi, il faut être attentif à cette éventualité et une étude particulière est nécessaire pour répondre à cette question.

Reçu le 18 août 1986. Accepté le 16 août 1987.

\section{RÉFÉRENCES BIBLIOGRAPHIQUES}

Abraham J., Simeone F. A., Hopkins R. W., 1976. A sensitive assay for allantoin. Anal. Biochem., 70, 377-380.

Domnas A., 1961. J. Biochem. (Tokyo), 50, 46-51, cité par Patterson T. G. et al., 1982.

Fosse R., Bossuyt V., 1927. Dosage de l'acide allantoïque à l'état de xanthylurée. Application à l'analyse des feuilles d'Acer pseudoplatanus. C. R. Acad. Sci. Paris, 185, 308-310.

Fosse R., Brunel A., Thomas P. E., 1931. Application de l'analyse quantitative spectrophotométrique de l'allantoine au sang de quelques mammifères et à la graine de nombreux végétaux. $C$. $R$. Acad. Sci. Paris, 193, 7-11.

Fosse R., Hieulle, 1924. C. R. Acad. Sci. Paris, 179, 636-638. Sur une réaction colorée supposée spécifique de l'aldéhyde formique produit par l'acide glyoxylique.

Jolivet E., Mossé J., 1982. Non-protein nitrogenous compounds with particular attention to ureides. In Aroba S. K., Chemistry and Biochemistry of Legumes, Oxford $18 \mathrm{H}$ Publ. Co, New-Delhi, 195216.

Jolivet E., Grandjean M., Monget C., Sauvage D., Lesaint C., 1985. Mise en évidence d'une nouvelle forme de transport de l'azote organique, l'acide allantö̈que, dans la sève racinaire du concombre. C. R. Acad. Agric. Fr., 71, 1369-1376.

Jolivet E., Lesaint C., Grandjean M., Monget C., Sauvage D., Roux L., 1986. Mise en évidence de voies différentes de synthèse des uréides glyoxyliques chez la tomate et le concombre par utilisation d'hypoxanthine, d'allopurinol et d'urée. C. R. Acad. Agri. Fr., 72, 429-437.

Kramer D. N., Klein N., Baselice R. A., 1959. Quantitative determination of glyoxylic acid. Anal. Chem., 31, 250-252.
Matsui M., Okada M., Ishidate M., 1965. Structure of the principal colored product formed in the determination of glyoxylic acid. Anal. Biochem., 12, 143-149.

Patterson T. G., Glenister R., La Rue T. A., 1982. Simple estimate of ureides in soybean tissue. Anal. Biochem., 119, 90-95.

Patton C. J., Crouch S. R., 1977. Spectrophotometric and kinetics investigation of the Berthelot reaction for the determination of ammonia. Anal. Biochem., 43, 464-469.

Thiemeyer W., Giesecke D., 1982. Quantitative determination of allantoin in biological fluids by reversed phase high-pressure liquid chromatography. Anal. Biochem., 123, 11-13.

Trijbels F., Vogels G. D., 1966. Degradation of allantoin by $P$ seu domonas acidovorans. Biochim. Biophys. Acta, 112, 292-301.

Van Berkum P., Sloger C., 1983. Analytical procedure for the determination of allantoin and allantoic acid in soybean tissue. Plant Physiol., 73, 511-513.

Van der Drift C., Vogels G. D., 1966. Allantoin and allantoate in higher plants. Acta Bot. Neerl., 15, 209-214.

Vogels G. D., Van der Drift C., 1970. Differential analyses of glyoxylate derivatives. Anal. Biochem., 33, 143-157.

Vrabski M. M., Grujic-Injac B., Gavic D., 1978. A new method for allantoin determination and its application in allantoin determination in Agrostemma githago L. seed. Anal. Biochem., 91, 304-308.

Young E. G., Conway F. C., 1942. On the estimation of allantoin by the Rimini-Schryver reaction. J. Biol. Chem., 142, 839-853.

Zengbé M., 1984. Contribution à l'étude du métabolisme des uréides chez le soja (Glycine max L. Merr.). Thèse d'Etat, Montpellier. 